\title{
Acute fibrinous and organizing pneumonia following hemophagocytic syndrome in two adult patients with hematological malignancies
}

\author{
Satoru Nanno, ${ }^{1)}$ Hideo Koh, ${ }^{1)}$ Hiroshi Okamura, ${ }^{1)}$ Mitsutaka Nishimoto, ${ }^{1)}$ Yasuhiro Nakashima, \\ Masahiko Ohsawa, ${ }^{2)}$ Masayuki Hino, ${ }^{1)}$ and Hirohisa Nakamae ${ }^{1)}$
}

Acute fibrinous and organizing pneumonia (AFOP) is a rare acute lung injury featuring pathological intra-alveolar fibrin balls and organizing pneumonia without hyaline membranes or eosinophils. AFOP forms acute and subacute patterns; the former often has a poor prognosis, whereas the latter has better survival. Secondary hemophagocytic syndrome (HPS) is a cytokinerelated and potentially lethal disorder induced by various diseases, and pulmonary involvement in HPS is not rare. However, to our knowledge, no report has addressed the association between secondary HPS and AFOP development. We report two cases of subacute AFOP following HPS in hematological malignancies.

Keywords: Acute fibrinous and organizing pneumonia, hemophagocytic syndrome, hematological malignancies

\section{INTRODUCTION}

Acute fibrinous and organizing pneumonia (AFOP), first described by Beasley in 2002, ${ }^{1}$ is a rare acute lung injury featuring pathological intra-alveolar fibrin balls and organizing pneumonia (OP) without hyaline membranes or eosinophils. AFOP presents acute and subacute clinical courses. The former often has a poor prognosis similar to diffuse alveolar damage (DAD), and the latter has a better prognosis similar to OP. ${ }^{1,2}$ Although the pathophysiology remains poorly understood, specific infections, connective tissue disorders, anticancer drugs, solid tumors, hematological malignancies, ${ }^{1-5}$ allogeneic hematopoietic stem cell transplantation, ${ }^{6}$ and lung transplantation ${ }^{7}$ may be related to AFOP development. On the other hand, hemophagocytic syndrome (HPS) is a well-known cytokine-related and potentially lethal disorder caused by abnormal immune activation of macrophages and cytotoxic T lymphocytes. ${ }^{8}$ Secondary HPS in adults is most commonly caused by underlying diseases, including infections, connective tissue disorders, and malignancies. The prognosis mainly depends on whether the underlying disease is responsive to treatment. ${ }^{8,9}$ In addition, HPS can be accompanied by pulmonary complications. ${ }^{10}$ However, to our knowledge, no report has addressed the association between HPS in hematological malignancies and AFOP development. We present two cases of subacute-type AFOP following HPS development in hematological malignancies.

\section{CASE REPORTS}

\section{Case 1}

In October 2009, a 63-year-old man was diagnosed with refractory anemia of myelodysplastic syndrome (MDS) related to trisomy 8 . One and a half years post-diagnosis, oral administration of low-dose prednisolone (PSL) and methenolone was initiated because he became transfusiondependent. Four months later, he was hospitalized because of high fever. On hospitalization day (HD) 1, he had pancytopenia (white blood cell count, 500/ $\mu \mathrm{L}$ [blasts, $8 \%$ ]; hemoglobin level, $7.3 \mathrm{~g} / \mathrm{dL}$; platelet count, $12.3 \times 10^{4} / \mu \mathrm{L}$ ), splenomegaly, and hyperferritinemia (serum ferritin level, 39,900 [normal, 21.8-274.7] ng/mL). Biochemistry examinations revealed an aspartate aminotransferase (AST) level of 51 (normal, 13-30) IU/L, alanine aminotransferase (ALT) level of 184 (normal, 6-27) IU/L, lactate dehydrogenase level of 363 (normal, 124-222) IU/L, and plasma fibrinogen level of 757 (normal, 200-400) mg/dL. Bone marrow aspiration

\footnotetext{
Received: August 25, 2020. Revised: January 27, 2021. Accepted: March 2, 2021. J-STAGE Advance Published: April 20, 2021 DOI: $10.3960 /$ jslrt.20042

${ }^{1)}$ Hematology, Graduate School of Medicine, Osaka City University, Osaka, Japan, ${ }^{2}$ Diagnostic pathology, Graduate School of Medicine, Osaka City University, Osaka, Japan Corresponding author: Hideo Koh, M.D., Ph.D., Hematology, Graduate School of Medicine, Osaka City University, 1-4-3 Asahi-machi, Abeno-ku, Osaka 545-8585, Japan.

E-mail: hide_koh@med.osaka-cu.ac.jp 
yielded a hypocellular smear with $22.4 \%$ blasts and $5.2 \%$ hemophagocytes, suggesting the progression to acute myeloblastic leukemia with suspected HPS. Chromosome analysis revealed a complex karyotype, including $5 \mathrm{q}$ deletion and trisomy 8. Although we were unable to assess the triglyceride level, soluble interleukin-2 receptor (sIL-2R) level, and natural killer (NK) activity, we diagnosed him with definitive HPS because he met five of the eight factors comprising the hemophagocytic lymphohistiocytosis (HLH) 2004 criteria. $^{9}$ In addition, chest X-ray and computed tomography (CT) demonstrated infiltration in the right middle lobe and multiple micronodules in both lungs (Figure 1A and 1B). Although serum galactomannan and $\beta$-D-glucan were negative, fungal pneumonia was suspected based on the imaging findings. He was intravenously treated using micafungin and tazobactam/piperacillin before treatment for MDS with HPS. These were subsequently switched to liposomal amphotericin B (LAMB) and meropenem due to refractoriness of the disease. However, his symptoms did not improve. We performed transbronchial lung biopsy of the right-sided infiltrative lesion and the diagnosis of AFOP was made on HD9 (Figure $1 \mathrm{C}$ and 1D). Although we performed culture of bacteria, fungi, and tuberculosis, in addition to Grocott-Gomori staining, we found no evidence of infection. Moreover, there was no histopathological evidence of other malignancies.
We administered methylprednisolone (mPSL) pulse therapy ( $1 \mathrm{~g} /$ body) for 3 days, followed by PSL maintenance therapy. Although the infiltrative shadow disappeared immediately, the micronodules increased in size. These lesions were diagnosed as probable invasive aspergillosis based on the high serum galactomannan levels (1.8 [normal, 0.5 ] cut-off index) on HD23. After LAMB was switched to voriconazole, the lesions gradually became smaller and disappeared. Although the signs and symptoms of HPS also improved, pancytopenia and hyperferritinemia persisted. Thereafter, he underwent chemotherapy followed by cord blood transplantation for refractory acute myeloid leukemia 7 months after discharge. One month later, he died of primary disease in July 2012, without AFOP relapse.

\section{Case 2}

In June 2011, a 38-year-old woman was diagnosed with primary mediastinal B-cell lymphoma (PMBCL). She achieved a partial response (PR) after receiving eight courses of rituximab, cyclophosphamide, doxorubicin, vincristine, and PSL regimen. She received 50-Gy radiation to the mediastinum and consequently achieved a first complete response at 1 year post-diagnosis. One month later, she was diagnosed with relapsed PMBCL because of the enlargement of mediastinal lymph nodes. Although we administered
(A)

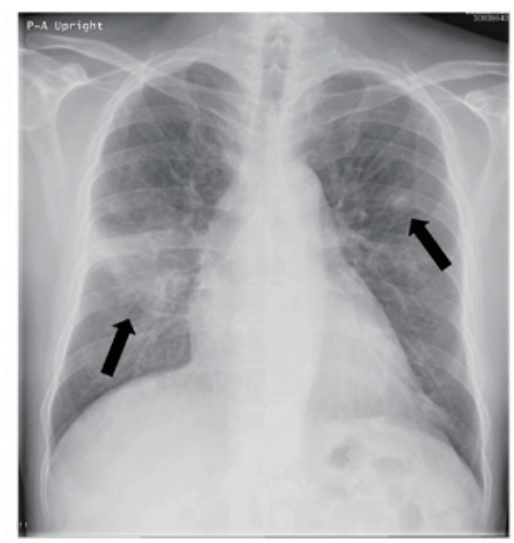

(C)

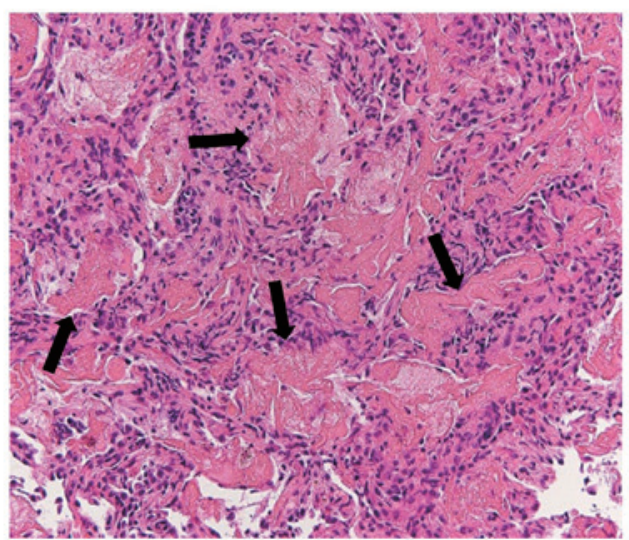

(B)

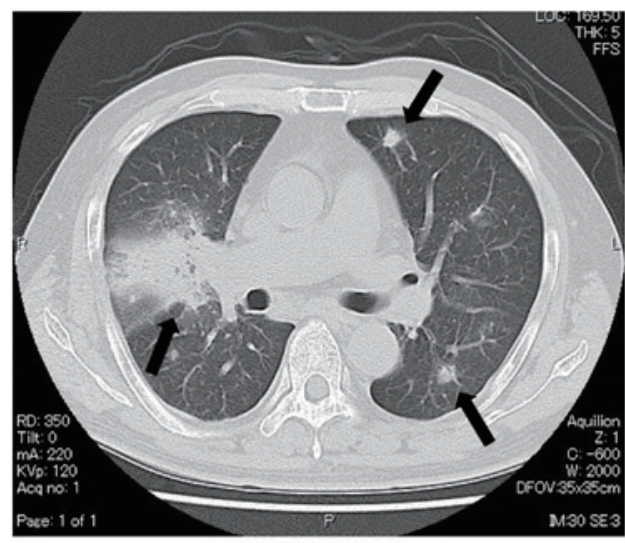

(D)

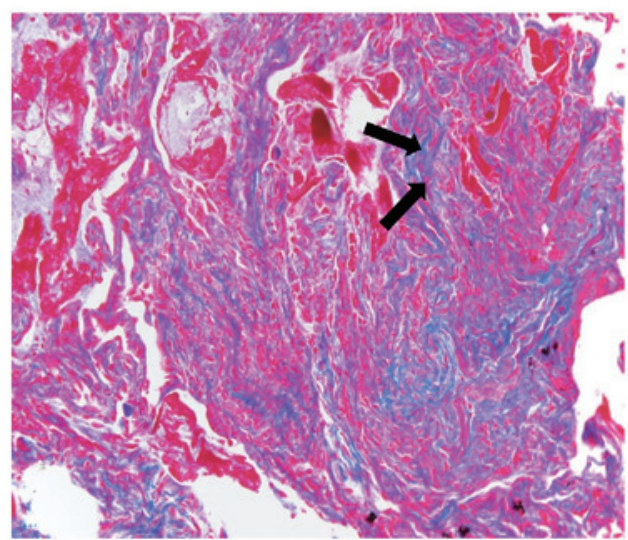

Fig. 1. $(\boldsymbol{A})$ Chest X-ray and $(\boldsymbol{B})$ computed tomography $(\mathrm{CT})$ demonstrated infiltration in the right middle lobe (arrow) and multiple micronodules (arrows) in the left lung. $(\boldsymbol{C})$ Hematoxylin and eosin staining showed intra-alveolar fibrin balls and organizing pneumonia without hyaline membranes or eosinophils (arrows) in the transbronchial biopsy specimen (original magnification $200 \times$ ). (D) Azan staining showed intra-alveolar fibrosis (arrows) (original magnification 100×) in Patient 1 at the onset of acute fibrinous and organizing pneumonia (AFOP) on hospital day 9. 
rituximab, etoposide, mPSL, high-dose cytarabine, and cisplatin, the treatment was not effective. We administered rituximab plus ranimustine, etoposide, cytarabine, and melphalan as the second salvage chemotherapy, and she achieved PR after receiving one course. On day (D) 7 of the second course, she exhibited pancytopenia (white blood cell count, $600 / \mu \mathrm{L}$; hemoglobin level, $10.0 \mathrm{~g} / \mathrm{dL}$; platelet count, $7.0 \times$ $104 / \mu \mathrm{L}$ ) and mildly high transaminase levels (AST and ALT levels of 34 and 76 IU/L, respectively). On D18, she developed febrile neutropenia. She was initially treated using intravenous cefepime, which was subsequently changed to imipenem/cilastatin, teicoplanin, and caspofungin. However, the symptoms did not improve. Chest CT revealed an infiltration shadow with ground-glass opacity (GGO) in the left side of the lungs on D34. Imipenem/cilastatin and caspofungin were changed to tazobactam/piperacillin and LAMB. As severe pancytopenia with fever remained, we performed bone marrow aspiration on D40. Bone marrow aspiration yielded a markedly hypocellular smear with $2.0 \%$ hemophagocytes and no lymphoma invasion. The chromosome karyotype was normal. Biochemistry testing demonstrated a serum ferritin level of $1916 \mathrm{ng} / \mathrm{mL}$, serum sIL-2R level of 2580 (normal, 124-466) IU/L, serum triglyceride level of 89 (normal, $50-150) \mathrm{mg} / \mathrm{dL}$, and plasma fibrinogen level of $639 \mathrm{mg} / \mathrm{dL}$. Although we were unable to evaluate NK activity and confirm splenomegaly, we diagnosed her with HPS because she

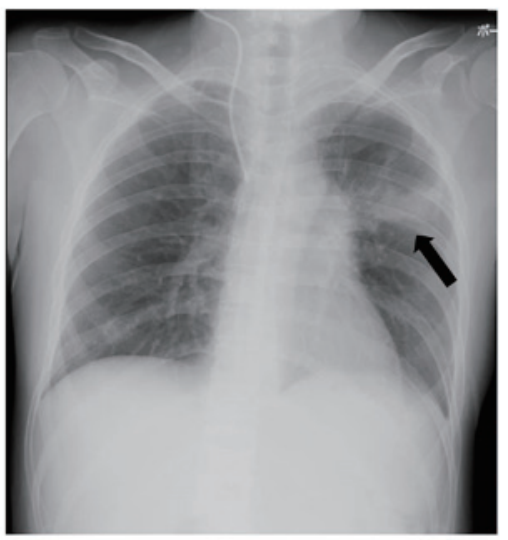

(C)

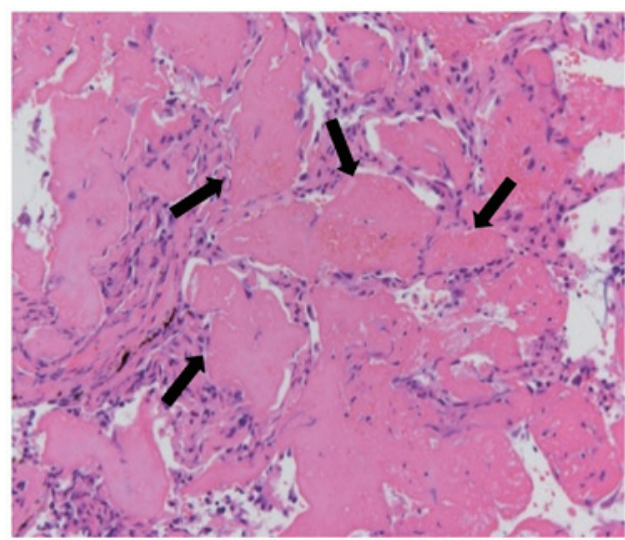

met five of the eight HLH 2004 criteria. $^{9}$ Thereafter, the infiltrative shadow with GGO gradually enlarged and a new GGO developed in the left side of the lungs (Figure 2A and 2B). We therefore performed transbronchial biopsy and diagnosed AFOP on D45 (Figure 2C and 2D). Although we performed culture of bacteria, fungi, and tuberculosis, in addition to Grocott-Gomori staining, we found no evidence of infection. Moreover, there was no histopathological evidence of other malignancies. After the initiation of PSL at $30 \mathrm{mg} /$ day intravenously, the infiltration shadow gradually disappeared and finally became a scar shadow. Although the signs and symptoms of HPS also improved, pancytopenia and hyperferritinemia persisted. Thereafter, she underwent allogeneic peripheral blood stem cell transplantation from a haploidentical sibling for PMBCL in PR because of poor autologous stem cell mobilization. At 1 year and 11 months post-diagnosis, she obtained complete response of the primary disease without AFOP relapse.

\section{DISCUSSION}

We reported two cases of subacute-type AFOP following HPS in hematological malignancies. The present two patients had pulmonary involvement in AFOP after the development of signs and symptoms of HPS. After the initiation of steroids, pulmonary involvement in AFOP immediately

(B)

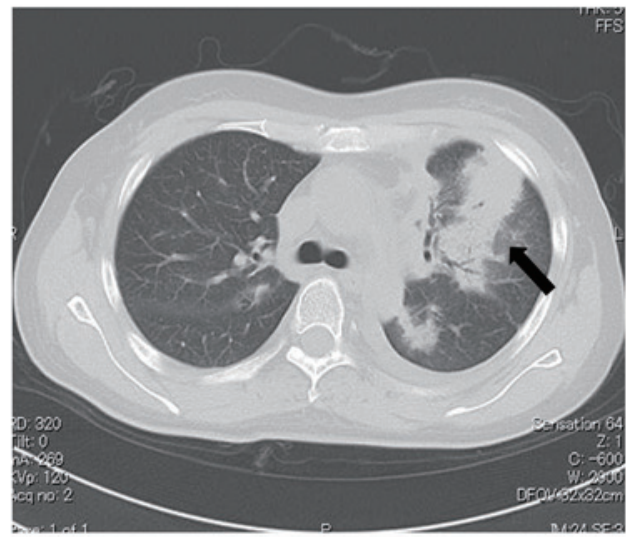

(D)

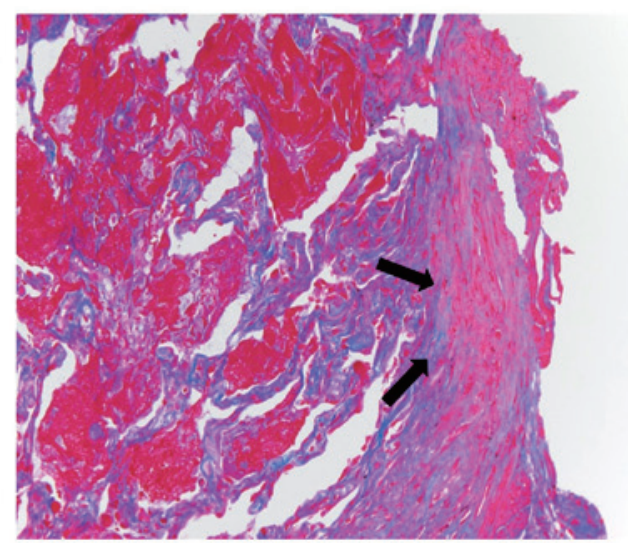

Fig. 2. (A) Chest X-ray and (B) CT demonstrated infiltration with ground-glass opacity (arrow) in the left lung. (C) Hematoxylin and eosin staining showed intra-alveolar fibrin balls and organizing pneumonia without hyaline membranes or eosinophils (arrows) in the transbronchial biopsy specimen (original magnification 200×). (D) Azan staining showed intra-alveolar fibrosis (arrows) (original magnification 100×) in Patient 2 at the onset of AFOP on day 45 of the second course of chemotherapy. 
disappeared, whereas hyperferritinemia and pancytopenia associated with HPS partially improved. In addition, although Patient 1 had simultaneous Aspergillus infection and AFOP, the meticulous observation of the response to steroids for AFOP led to identification of the nonresponsive lesions as Aspergillus infection.

Several case series and retrospective studies ${ }^{1,3-7,10}$ demonstrated nonspecific symptoms, such as fever and cough, and radiological findings, including predominantly lobar consolidation with and without GGO, in patients with AFOP. Although the treatment for AFOP remains to be established, steroids are predominantly used. The mortality of the acute type is greater than $50 \% .^{1,4}$ In contrast, 16 patients with the subacute type responded to steroids, except for two whose underlying diseases progressed. ${ }^{11}$ Our two patients had nonspecific symptoms and responded to steroids, consistent with previous reports. However, enlargement of the micronodules during PSL administration in Patient 1 was an atypical clinical course. Although we initially suspected the multiple nodules to be AFOP and the right middle lobe infiltration to be AFOP by TBLB, the nodules were finally confirmed to be probable invasive aspergillosis based on their refractoriness to steroids and good response to antifungals. We had difficulty differentiating between AFOP and probable invasive aspergillosis because multiple nodules were observed in AFOP. ${ }^{12}$ We should consider the possibility of concomitant infections, including Aspergillus, in patients with steroidresistant subacute-type AFOP.

The pathophysiology of AFOP also remains unclear. A few case reports suggested that the overexpression of heme oxygenase-1 (HO-1) induced by inflammatory cytokines, including tumor necrosis factor (TNF)- $\alpha$, i.e., oxidative stress, is associated with AFOP development. ${ }^{6,13}$ In addition, both TNF- $\alpha^{14}$ and HO- 1 levels ${ }^{15}$ increase in HPS. Based on these observations, HPS development may affect the development of AFOP, possibly through mechanisms related to TNF- $\alpha$ and HO-1. Furthermore, Gomes et al. demonstrated that the incidence of AFOP was higher in the population with hematological malignancies. ${ }^{4}$ They hypothesized that patients with hematological malignancies have a certain level of risk for opportunistic infections, which may lead to AFOP development. In Patient 1, this and the mechanism mentioned above may have been involved in AFOP development. Further studies are warranted to explore the mechanisms of AFOP development.

In conclusion, we reported two cases of subacute-type AFOP following HPS development in hematological malignancies. In cases of subacute-type AFOP with HPS, steroid therapy may be effective for AFOP regardless of its response to HPS. Further investigation is required to elucidate the pathophysiology of AFOP related to HPS.

\section{CONFLICT OF INTEREST}

The authors state that they have no conflicts of interest .

\section{REFERENCES}

1 Beasley MB, Franks TJ, Galvin JR, Gochuico B, Travis WD. Acute fibrinous and organizing pneumonia: a histological pattern of lung injury and possible variant of diffuse alveolar damage. Arch Pathol Lab Med. 2002; 126 : 1064-1070.

2 Travis WD, Costabel U, Hansell DM, et al. An official American Thoracic Society/European Respiratory Society statement: Update of the international multidisciplinary classification of the idiopathic interstitial pneumonias. Am J Respir Crit Care Med. 2013; $188:$ 733-748.

3 Feinstein MB, DeSouza SA, Moreira AL, et al. A comparison of the pathological, clinical and radiographical, features of cryptogenic organising pneumonia, acute fibrinous and organising pneumonia and granulomatous organising pneumonia. J Clin Pathol. 2015; 68 : 441-447.

4 Gomes R, Padrão E, Dabó H, et al. Acute fibrinous and organizing pneumonia: A report of 13 cases in a tertiary university hospital. Medicine (Baltimore). 2016; 95 : e4073.

5 Yamamoto M, Murata K, Kiriu T, Kouzai Y, Takamori M. Acute fibrinous and organizing pneumonia with myelodysplastic syndrome: corticosteroid monotherapy led to successful ventilator weaning. Intern Med. 2016; $55:$ 3155-3159.

6 Simmons GL, Chung HM, McCarty JM, et al. Treatment of acute fibrinous organizing pneumonia following hematopoietic cell transplantation with etanercept. Bone Marrow Transplant. 2017; 52 : 141-143.

7 Paraskeva M, McLean C, Ellis S, et al. Acute fibrinoid organizing pneumonia after lung transplantation. Am J Respir Crit Care Med. 2013; $187:$ 1360-1368.

8 Henter JI, Horne A, Aricó M, et al. HLH-2004: Diagnostic and therapeutic guidelines for hemophagocytic lymphohistiocytosis. Pediatr Blood Cancer. 2007; 48 : 124-131.

9 La Rosée P, Horne A, Hines M, et al. Recommendations for the management of hemophagocytic lymphohistiocytosis in adults. Blood. 2019; 133 : 2465-2477.

10 Seguin A, Galicier L, Boutboul D, Lemiale V, Azoulay E. Pulmonary involvement in patients with hemophagocytic lymphohistiocytosis. Chest. 2016; 149 : 1294-1301.

11 Dai JH, Li H, Shen W, et al. Clinical and radiological profile of acute fibrinous and organizing pneumonia: a retrospective study. Chin Med J (Engl). 2015; 128 : 2701-2706.

12 Labarinas S, Gumy-Pause F, Rougemont AL, et al. Is acute fibrinous and organizing pneumonia the expression of immune dysregulation? J Pediatr Hematol Oncol. 2013; 35 : 139-143.

13 Hara Y, Shinkai M, Kanoh S, et al. Clinico-pathological analysis referring hemeoxygenase- 1 in acute fibrinous and organizing pneumonia patients. Respir Med Case Rep. 2015; 14 : 53-56.

14 Vick EJ, Patel K, Prouet P, Martin MG. Proliferation through activation: hemophagocytic lymphohistiocytosis in hematologic malignancy. Blood Adv. 2017; 1 : 779-791.

15 Miyazaki T, Kirino Y, Takeno M, et al. Serum HO-1 is useful to make differential diagnosis of secondary hemophagocytic syndrome from other similar hematological conditions. Int J Hematol. 2010; 91 : 229-237. 